\title{
The growth rate of cosmic structures in the local Universe with the ALFALFA survey
}

\author{
F. Avila,${ }^{1 \star}$ A. Bernui ${ }^{1}$ E. de Carvalho, ${ }^{2}$ C. P. Novaes ${ }^{3}$ \\ ${ }^{1}$ Observatório Nacional, Rua General José Cristino 77, São Cristóvão, 20921-400 Rio de Janeiro, RJ, Brazil \\ ${ }^{2}$ Centro de Estudos Superiores de Tabatinga, Universidade do Estado do Amazonas, 69640-000, Tabatinga, AM, Brazil \\ ${ }^{3}$ Instituto Nacional de Pesquisas Espaciais, Av. dos Astronautas 1758, Jardim da Granja, São José dos Campos, SP, Brazil
}

\section{Accepted XXX. Received YYY; in original form ZZZ}

\begin{abstract}
We investigate the growth rate of structures in the local Universe. For this, we use as a cosmological tracer the HI line extra-galactic sources from the Arecibo Legacy Fast ALFA (ALFALFA) survey to obtain a measurement of the normalized growth rate parameter, $f \sigma_{8}$, considered a powerful tool to constrain alternative models of gravity. For these analyses, we calculate the Local Group velocity due to the matter structures distribution in the ALFALFA catalogue and compare it with the Local Group velocity relative to the Cosmic Microwave Background frame to obtain the velocity scale parameter, $\beta$. Using Monte Carlo realizations and log-normal simulations, our methodology quantifies the errors introduced by shot-noise and partial sky coverage of the analysed data. The measurement of the velocity scale parameter $\beta$, and the calculation of the matter fluctuation of the cosmological tracer, $\sigma_{8}^{\mathrm{tr}}$, lead us to $f \sigma_{8}=0.46 \pm 0.06$ at $\bar{z}=0.013$, in good agreement (at $1 \sigma$ level) with the value expected in the $\Lambda$ CDM concordance model. In addition, our analyses of the ALFALFA sample also provide a measurement of the growth rate of structures $f=0.56 \pm 0.07$, at $\bar{z}=0.013$.
\end{abstract}

Key words: Cosmology: Observations - Cosmology: Large-Scale Structure of the Universe - galaxies: Local Group

\section{INTRODUCTION}

The large-scale structure data from current astronomical surveys contain the imprints of matter clustering evolution caused by gravitational instability (Pezzotta et al. 2017; Bautista et al. 2018; Haude et al. 2019; Marques \& Bernui 2020; Aubert et al. 2020). How these instabilities evolve over time is a crucial test for theories that aim to understand possible deviations of cosmological models based on general relativity (GR). The growth rate of cosmic structures, $f(a)$, is defined as (Strauss \& Willick 1995)

$f(a) \equiv \frac{d \ln D(a)}{d \ln a}$

where $D=D(a)$ is the linear growth function, and $a$ is the scale factor in the Robertson-Walker metric based on GR theory. In fact, $f=f(a)$ has the potential to constrain alternative models of gravity and dark energy from the measure of the growth index, $\gamma$, when one parametrizes $f$ as (Linder \& Cahn 2007)

$f(a)=\Omega_{m}^{\gamma}$.

In the $\Lambda \mathrm{CDM}$ model, based on GR, $\gamma \simeq 0.55$, and for some $f(R)$ modified gravity models $\gamma \simeq 0.41-0.21 z$ (Basilakos 2012).

The literature reports the analyses of several cosmological probes used to constrain $f(a)$, like the Cosmic Microwave

* e-mail: felipeavila@on.br
Background (CMB) (Planck Collaboration 2020), cluster abundances (Planck Collaboration 2016), weak lensing (Abbott et al. 2018), redshift space distortions (RSD) (Alam et al. 2017), and peculiar velocities (Boruah, Hudson \& Lavaux 2019), among others. Measurements of the growth rate, as well as other cosmological observables, are useful to determine the best parameters of the current cosmological model (de Carvalho et al. 2018; Nunes \& Bernui 2020).

Although some studies intend to measure $f(a)$ at some redshift, in practice they also constrain other parameters. For instance, the approach with peculiar velocities constrains the velocity scale parameter $\beta \equiv f / b$, where $b$ is the linear bias defined by $b \equiv \sigma_{8}^{\text {tr }} / \sigma_{8}^{\mathrm{m}}$ (Papageorgiou et al. 2012), $\sigma_{8}^{m}$ is the matter fluctuation at radius $8 \mathrm{Mpc} / h$ (hereafter $\sigma_{8} \equiv \sigma_{8}^{m}$ ) and $\sigma_{8}^{\mathrm{tr}}$ is the matter fluctuation of the cosmological tracer (e.g., blue galaxies, luminous red galaxies, etc.) at radius $8 \mathrm{Mpc} / \mathrm{h}$. The most common constraint found in the literature is the combination $f \sigma_{8}$. An interesting approach to obtain $f \sigma_{8}$ is to measure first $\beta$, and then find $f \sigma_{8}=\beta b \sigma_{8}$, or equivalently

$f \sigma_{8}=\beta \sigma_{8}^{\mathrm{tr}}$.

For a local Universe sample, $z \lesssim 0.1$, the gravitational dipole approach is suitable to measure $\beta$ and, consequently, $f \sigma_{8}$, using the equation (3) (see, e.g., Strauss \& Willick (1995); Scaramella, Vettolani \& Zamorani (1994)). If one compares the peculiar veloc- 
ity of our Local Group (LG) of galaxies inferred from the CMB dipole, $627 \pm 22 \mathrm{~km} / \mathrm{s}$ towards $(l, b)=\left(273^{\circ} \pm 3^{\circ}, 29^{\circ} \pm\right.$ $3^{\circ}$ ) (Kogut et al. 1993; Courteau \& Van Den Bergh 1999; Erdoğdu et al. 2006), with the value measured in a local survey, one can constrain $\beta$. For example, in the analyses done by Erdoğdu et al. (2006), they used the 2 Micron All-sky Redshift Survey (2MRS) to estimate $\beta=0.40 \pm 0.09$, with the dipole converging to a constant value around $60 \mathrm{Mpc} / \mathrm{h}$. In another work, Basilakos \& Plionis (2006) re-examined the Point Source catalogue redshift (PSCz) survey (Rowan-Robinson et al. 2000) estimating the $\beta$ parameter as $\beta \simeq 0.49$, with significantly contribution to the dipole magnitude from distances beyond $185 \mathrm{Mpc} / h$. In addition, using a fullsky X-ray cluster sample, Kocevski \& Ebeling (2006) calculated $\beta=0.24 \pm 0.01$ using the number-weighted method (see Section 3.1). These authors also observe contributions to the dipole velocity beyond $185 \mathrm{Mpc} / \mathrm{h}$. As reported by Bilicki et al. (2011), one should not expect consistency between different analyses of diverse cosmic tracers for the amplitude and the scale of convergence. Regarding $\beta$, the $\Lambda \mathrm{CDM}$ model is a good guide to find out if the result found for $\beta$ is in any way consistent, that is, $\beta=\Omega_{m}^{\Lambda \mathrm{CDM}}(z)^{0.55} / b$. Despite the differences found in the literature about the convergence scale, that is, the minimum scale where the dipole velocity attains its stability value (Kocevski \& Ebeling 2006), we will show, for the sample in analysis, that the magnitude of the gravitational dipole is reached around $60 \mathrm{Mpc}$.

In this work we investigate the clustering of the extra-galactic HI line sources observed in the, recently completed, ALFALFA Survey (Haynes et al. 2018) to perform a measurement of $f \sigma_{8}$ in the local Universe, i.e., at $\bar{z}=0.013$. Although the ALFALFA catalogue does not contain full-sky data, since the surveyed area is $\Omega \simeq 6900 \operatorname{deg}^{2}\left(f_{\text {sky }} \simeq 1 / 6\right)$, a distinctive feature is that it has a high number density of objects compared with full-sky catalogues in the same redshift range. This reduces the shot noise and increases the efficiency of the selection function, ruling out artificial convergences in the dipole magnitude, a crucial property for the approach we adopt here, and that we shall explain below. Another important attribute of the ALFALFA catalogue is that for the data sample with $c z_{\odot}<6000 \mathrm{~km} / \mathrm{s}$ the dipole can be calculated in the real space avoiding the RSD effect, known as the rocket effect (Kaiser 1987; Kaiser \& Lahav 1989). Regarding the error and systematic sources, our analyses take into account the incomplete sky coverage by using log-normal simulations (Agrawal et al. 2017), that help us to correct both direction and magnitude of the gravitational dipole (also called clustering dipole), and a set of Monte Carlo realizations, proposed by Basilakos \& Plionis (1998) to estimate the shot-noise error, procedures described in Section 3.2 (for diverse analyses regarding systematics see, e.g., Marques et al. 2018; Avila et al. 2018, 2019; de Carvalho et al. 2020, 2021; Sarkar \& Pandey 2019; Pandey \& Sarkar 2020; Heinesen 2020).

The work is structured as follows. The ALFALFA catalogue is presented in Section 2, together with the selection function calculation and the criteria to select the final data sample for analysis. In Section 3 we detail the methodology to calculate the LG dipole, their error estimation and the $\sigma_{8}^{\mathrm{tr}}$ calculation. The results of our analyses and our conclusions are presented in Sections 4 and 5, respectively.

\section{THE ARECIBO LEGACY FAST ALFA SURVEY}

The Arecibo Legacy Fast ALFA Survey ${ }^{1}$ (ALFALFA; Haynes et al. 2011, 2018) was a blind $21 \mathrm{~cm} \mathrm{HI}$ line survey designed with the main goal of obtaining a robust measurement of the HI mass function, an important component, together with the luminosity function, that can make a significant contribution to the study of galaxy population in the local Universe (Giovanelli \& Haynes 2016; Jones et al. 2016, 2018, 2020). Given the surveyed area and the spectral resolution, ALFALFA can measure the faint end of the $\mathrm{HI}$ mass function for the optically faint, gas-rich population (O'Donoghue 2018). For additional studies see Haynes et al. (2018) and references therein.

The ALFALFA survey was performed between 2005 and 2011 covering an area of $\Omega \simeq 6900 \mathrm{deg}^{2}$ out to $z<0.06$ detecting $31500 \mathrm{HI}$ line extra-galactic sources. The survey covers two discontinuous regions, both in the declination range of $0^{\circ}<$ DEC $<$ $36^{\circ}$, in the right ascension intervals of $21^{\mathrm{h}} 30^{\mathrm{m}}<\mathrm{RA}<3^{\mathrm{h}} 15^{\mathrm{m}}$ (South galactic hemisphere) and $7^{\mathrm{h}} 20^{\mathrm{m}}<\mathrm{RA}<16^{\mathrm{h}} 40^{\mathrm{m}}$ (North galactic hemisphere). The catalogue distinguish the sources with a CODE 1,2, and 9, according to quality of the data observed (Haynes et al. 2018). CODE 1, refers to a high signal-to-noise ratio detection of the HI extragalactic source, with confirmed optical counterpart; CODE 2, lower signal to noise ratio coincident with optical counterpart, they are considered unreliable sources; and CODE 9, high signal to noise ratio source with no optical counterpart and likely Galactic high velocity cloud. In this work we shall use only the sources with CODE 1, as recommended by the ALFALFA team.

\subsection{Data selection}

The distances data presented in the ALFALFA catalogue are described in Haynes et al. (2018) (see Section 3.1, column 11). The ALFALFA collaboration uses two distances estimation approaches: (i) for those objects with $c z_{\odot}>6000 \mathrm{~km} / \mathrm{s}$ the distance is simply estimated as $c z_{\mathrm{CMB}} / H_{0}$, where $c z_{c m b}$ is the recessional velocity measured in the Cosmic Microwave Background reference frame and $H_{0}$ is the Hubble constant; and, (ii) for objects with $c z_{\mathrm{CMB}}<6000 \mathrm{~km} / \mathrm{s}$ the collaboration assigns distances to nearby galaxies through a parametric flow model developed by Master (2005), based mainly on the SFI++ catalogue of galaxies (Springob et al. 2007) and results from analysis of the peculiar motion of galaxies, groups, and clusters, using a combination of primary distances from the literature and secondary distances from the TullyFisher relation. Also, when available, they use known distances from the literature. In Appendix A we test the impact of distance uncertainties on dipole analyses.

The transition velocity between the methodologies applied to calculate distances, that is, $6000 \mathrm{~km} / \mathrm{s}$, means $85-90 \mathrm{Mpc}$, and this interval corresponds to the discontinuity observed in the HubbleLemaître diagram, shown in Fig. 1. Due to this fact, we applied a conservative cut to remove the HI sources with distances above $85 \mathrm{Mpc}$ (the red vertical line in Fig. 1 illustrates this cut). In this way we are sure that all objects from the sample selected for our dipole analyses have all their distance measurements performed using only one methodology. As also observed in Fig. 1, some objects appear far from the Hubble flow, probably due to their intense peculiar motions. This systematic effect motivates an examination.

1 http://egg.astro.cornell.edu/alfalfa/data/index. php 
For this, in Appendix B, we performed a comparative test with and without the outlier HI sources.

The next constraint applied to the sample concerns the presence of the Virgo cluster. We observe that it is composed by 224 HI line sources, a potential source of systematics. In fact, the radial distances attributed to the members of the Virgo cluster in the ALFALFA catalogue is not realistic, as observed in Fig. 2 (see discussions about the adopted distances in Haynes et al. 2018). The discontinuous distribution of distances of the galaxy Virgo members, clearly observed in Fig. 2, is due to the lack of information regarding the distance of each galaxy to us. For this, the distances of the galaxies inside Virgo are only assigned by identifying the groups or substructures and assigning the same distance to all their members. Therefore, to avoid biasing our calculations, we remove the Virgo members from our sample.

Our final restriction aims to avoid the contribution of LG member galaxies, which should not be accounted for the dipole calculation. Therefore, since we are considering the LG as an unique structure, we follow Bilicki et al. (2011); Bilicki (2012); Erdoğdu et al. (2006) and remove these galaxies. We assume the LG as a spherical structure of $1.5 \mathrm{Mpc}$ radius centred near us, as suggested by observations (Van den Bergh 2000; Van der Marel \& Guhathakurta 2008). Then, we localize and remove from our catalogue 8 HI line sources corresponding to this region.

After all these cuts the final data sample for analyses contains $N=7798 \mathrm{HI}$ line extra-galactic sources, with median redshift $\bar{z}=0.013$, and number density $\bar{n}=0.04 \mathrm{Mpc}^{-3}$. In Fig. 3 we show the distribution of their distances, and in Fig. 4 their Aitoff projection on the celestial sphere in galactic coordinates.

Two important features of this final data sample regarding our study of the gravitational dipole deserve some comments: (i) it does not cover the full sky, and (ii) it does not cover the area corresponding to the LG peculiar motion direction inferred from the CMB dipole, as shown in Fig. 4 as a blue triangle. With respect to the first feature, our methodology to estimate the error for $f \sigma_{8}$ takes into account the incomplete sky coverage, as described in the Section 3.2. Regarding the second feature, one does not expect that with a partial sky coverage of the data sample one could find the LG dipole direction aligned, or close to, the CMB dipole direction; fortunately, our interest is just in the modulus part of the LG dipole, which is affected by the partial coverage of the sky, but can be corrected by evaluating the impact of such feature through a set of simulated data, as we shall see.

\subsection{The radial selection function}

In order to calculate the gravitational dipole we must assign a weight $\omega_{i}$ for the $i$ th galaxy (Scaramella, Vettolani \& Zamorani 1994)

$\omega_{i}=\frac{1}{\phi\left(r_{i}\right)}$,

where $\phi\left(r_{i}\right)$ is the radial selection function. To calculate $\phi(r)$ for the ALFALFA sample we must obtain the two-dimensional number density distribution, $n\left(m_{\mathrm{HI}}, \omega_{50}\right)$, where $m_{\mathrm{HI}} \equiv \log \left(M_{\mathrm{HI}}\right)$ and $M_{\mathrm{HI}}$ is the HI mass, measured in solar mass units, and $w_{50} \equiv$ $\log \left(W_{50}\right)$ is associated to the velocity width of the HI spectrum, $W_{50}$, measured at the $50 \%$ level, as described in Haynes et al. (2018), in units of $\mathrm{km} / \mathrm{s}$. Then, $\phi(r)$ is calculated as (Papastergis

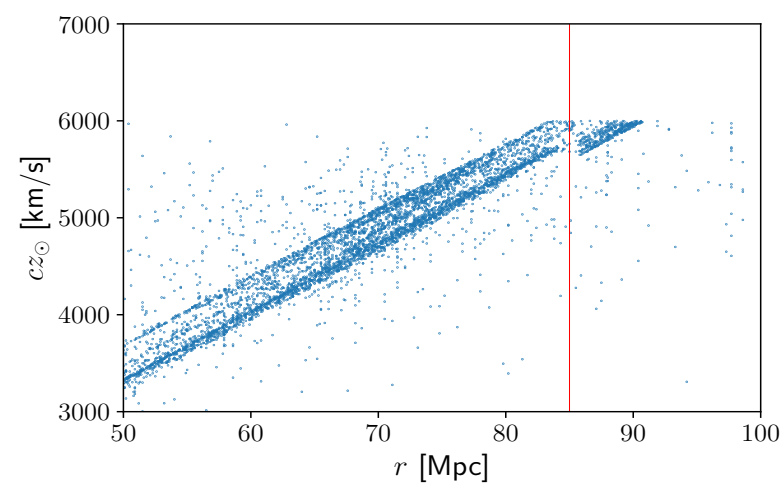

Figure 1. The Hubble-Lemaître diagram for the ALFALFA HI line sources below $c z_{\odot}<6000 \mathrm{~km} / \mathrm{s}$ and with CODE 1 . Notice the discontinuity around $r=85 \mathrm{Mpc}$, which appears because the ALFALFA team adopted a different approach to determine the distances. For this, we decided to remove from our sample those objects at distances larger than $85 \mathrm{Mpc}$.

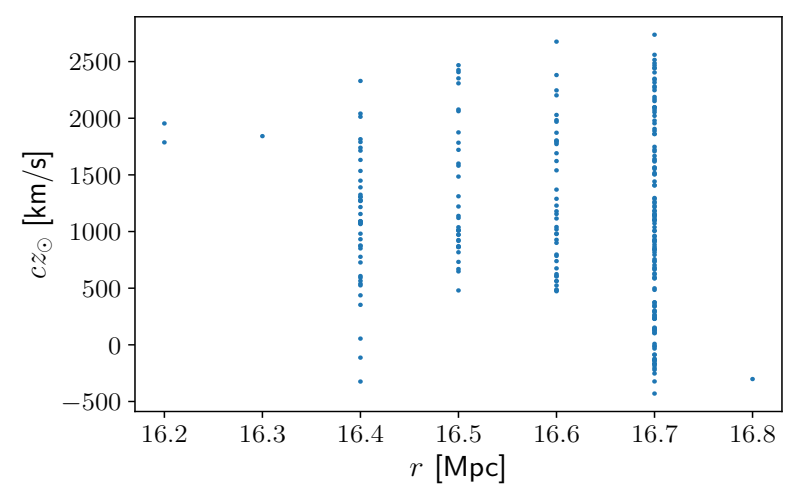

Figure 2. Distribution of distances versus velocities (i.e., the HubbleLemaitre plot) for the members of the Virgo cluster. One observes various discontinuities, evidencing the difficulty of the ALFALFA survey to determine the individual distances of the Virgo cluster galaxies, suggestive of non-linear dynamics that can bias the analyses. These objects were removed from the sample in analysis.

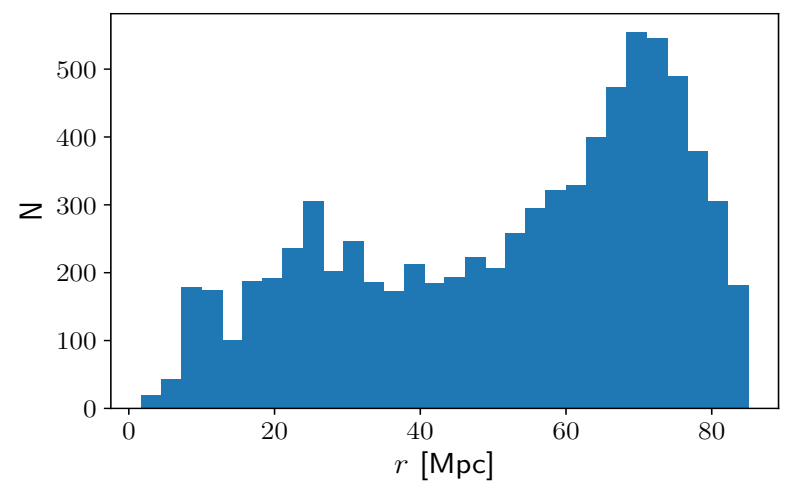

Figure 3. Histogram of the distances distribution of the ALFALFA HI line sources of the final data sample used in this work. See Section 2.1 for more information on how the sample was selected. 


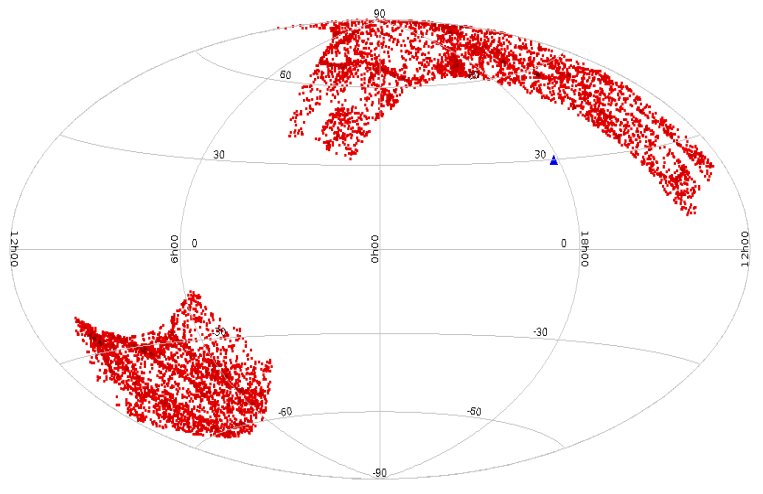

Figure 4. The HI line sources of our final data sample in the galactic Aitoff projection. The blue triangle indicates the direction of the LG velocity $(l, b)=\left(273^{\circ} \pm 3^{\circ}, 29^{\circ} \pm 3^{\circ}\right)$ inferred using the CMB dipole measurement.

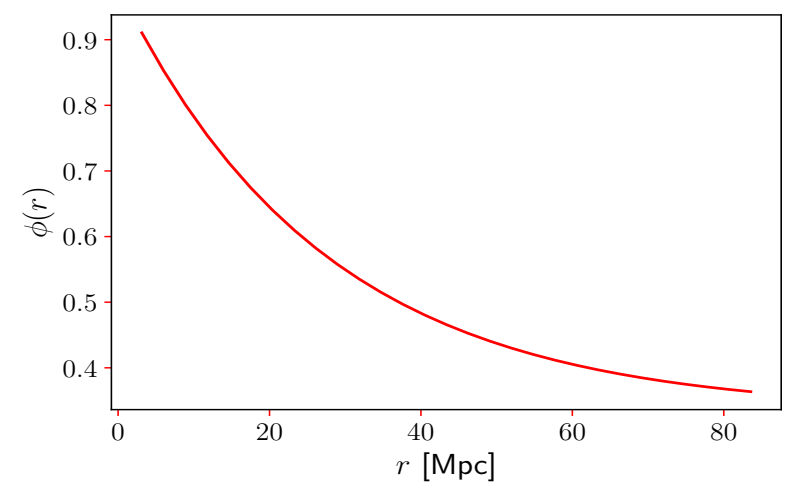

Figure 5. Radial selection function for the data in analysis. For details, see Section 2.2 .

et al. 2013)

$\phi(r)=\frac{\int_{\omega_{i}}^{\omega_{f}} \int_{m(r)}^{m_{f}} n(m, \omega) d m d \omega}{\int_{\omega_{i}}^{\omega_{f}} \int_{m_{i}}^{m_{f}} n(m, \omega) d m d \omega}$.

We dropped the sub-indices $\mathrm{HI}$ and 50 for simplicity. Notice that the integral in mass in the numerator uses as lower limit the minimum $\mathrm{HI}$ mass detectable at a distance $r, m_{\mathrm{HI}}(r)$. The other integrals are performed over the whole range of mass $m_{i} \leqslant m_{\mathrm{HI}} \leqslant$ $m_{f}$, and velocity width, $\omega_{i} \leqslant \omega_{50} \leqslant \omega_{f}$, of the objects in the ALFALFA sample. In Fig. 5 we show the result of this calculation, that is, the radial selection function for our data analyses.

\section{METHODOLOGY}

In this section we discuss the number-weighted method, a procedure to measure the LG velocity, caused by large-scale matter distribution, and the $\beta$ parameter, an intermediary step to obtain $f \sigma_{8}$. To apply this methodology one assumes the data to be a representative sample of the local Universe, besides that the galaxy clustering can be analysed using linear theory of perturbations. In what follows, we also describe the error estimations, that is, the contribution from shot-noise and the incomplete sky coverage, and the necessary correction. Lastly, we show the way to measure $\sigma_{8}^{\text {tr }}$ to obtain $f \sigma_{8}$ from equation (3).

\subsection{Number-weighted method and the LG dipole}

From the linear theory of gravitational instability, the peculiar velocity field $\mathbf{v}$ is related to the gravitational field, $\mathbf{g}$, by (Peebles 1980)

$\mathbf{v}(\mathbf{r})=\frac{H_{0} f}{4 \pi G \bar{\rho}} \mathbf{g}(\mathbf{r})$

where $f$ is the growth rate defined in equation (1), $H_{0}$ is the Hubble constant measured today, $G$ is the gravitational constant, and $\bar{\rho}$ is the matter density averaged over a large volume $V$. If we write $\mathbf{g}$ as

$\mathbf{g}(\mathbf{r})=G \bar{\rho} \int_{V} \delta_{\mathrm{m}}\left(\mathbf{r}^{\prime}\right) \frac{\mathbf{r}^{\prime}-\mathbf{r}}{\left|\mathbf{r}^{\prime}-\mathbf{r}\right|^{3}} d^{3} \mathbf{r}^{\prime}$

one can calculate the peculiar velocity if one knows the matter density contrast, $\delta_{\mathrm{m}}(\mathbf{r})$.

To measure $\mathbf{g}$ for a galaxy survey, the linear relation between matter and tracer contrasts can be assumed,

$\delta_{\mathrm{tr}}=b \delta_{\mathrm{m}}$,

where $b$ is the linear bias, and we consider the LG barycentre as the origin of coordinates, that is $\mathbf{r}=\mathbf{0}$. If the survey volume is large enough to ensure the convergence of the integral (Scaramella, Vettolani \& Zamorani 1994), one can rewrite equation (6) as

$\mathbf{v}_{\mathrm{LG}}(r)=\frac{H_{0} \beta}{4 \pi \bar{n}} \mathbf{D}_{\mathrm{LG}}(r)$,

where the LG clustering dipole, $\mathbf{D}_{\mathrm{LG}}$, is defined by

$\mathbf{D}_{\mathrm{LG}}(r) \equiv \sum_{i}^{N(r)} \frac{\hat{\mathbf{r}}_{i}}{\phi\left(r_{i}\right) r_{i}^{2}}$,

summing over all objects inside the sphere of radius $r$. Equation (9) is known as the number-weighted method (Erdoğdu et al. 2006).

\subsection{Error estimation}

In order to correctly obtain $\beta$, several effects that can influence the gravitational dipole magnitude must be taken into account (Schmoldt et al. 1999). As we already mentioned, one of them is the RSD effect, that can be avoided here because the distances of our ALFALFA sample are provided in real space. On the other hand, we must also evaluate the error introduced by shotnoise and the impact of using an incomplete sky coverage. Following, we discuss the methodology used to take into account each of them.

\subsubsection{Shot-Noise}

Following Basilakos \& Plionis (1998), we use a set of $N_{\mathrm{MC}}=144$ Monte Carlo realizations to calculate the error due to the shot-noise. This number of Monte Carlos is to equalize the number of lognormal simulations, $N=144$, used to correct the dipole velocity due to the partial sky coverage of our data sample (see Section 3.2.2 for details).

The methodology to produce each realization is to randomize the angular coordinates of the sources, i.e., taking their right ascension and declination from uniform random distributions limited by the ALFALFA footprint, repositioning them inside the same region, while their distances remain the same, keeping unchanged the selection function. From the set of Monte Carlo realizations, one can 
calculate the covariance matrix due to the shot-noise as

$$
\begin{aligned}
C_{\mathrm{SN}}^{i j}= & \frac{H_{0}}{4 \pi \bar{n}} \frac{1}{N-1} \sum_{k=1}^{N}\left[D_{\mathrm{LG}}^{k}\left(r_{i}\right)-\left\langle D_{\mathrm{LG}}\right\rangle\left(r_{i}\right)\right] \times \\
& {\left[D_{\mathrm{LG}}^{k}\left(r_{j}\right)-\left\langle D_{\mathrm{LG}}\right\rangle\left(r_{j}\right)\right], }
\end{aligned}
$$

where $D_{\mathrm{LG}}^{k} \equiv\left|\mathbf{D}_{\mathrm{LG}}^{k}\right|$ is the dipole magnitude calculated for the $k$ th Monte Carlo realization and $\left\langle D_{\mathrm{LG}}\right\rangle$ is the average dipole over all the $N_{\mathrm{MC}}=144$ Monte Carlo realizations.

\subsubsection{Correction procedure for incomplete sky coverage}

The incomplete sky coverage of the sample in analysis, shown in Fig. 4, certainly bias the measurement of direction and magnitude of the LG dipole velocity, which must be corrected accordingly. The idea is to use simulated catalogues to perform both, full and partial sky dipole analyses, that allow to find the corrected LG dipole velocity.

Consider a set of $N$ full-sky (FS) log-normal simulated catalogues, to which we apply the ALFALFA footprint to obtain the respective partial sky (PS) log-normal simulated catalogues. Then, we measure the dipoles of the $i$ th catalogue, $\mathbf{D}_{\text {sim }}^{\mathrm{FS}, i}$ and $\mathbf{D}_{\text {sim }}^{\mathrm{PS}, i}$, for $i=1, \cdots, N$, for the FS and PS cases, respectively. The difference, $\mathbf{X}^{i}(r)$, defined as

$\mathbf{X}^{i}(r) \equiv \mathbf{D}_{\text {sim }}^{\mathrm{FS}, i}(r)-\mathbf{D}_{\text {sim }}^{\mathrm{PS}, i}(r)$,

is used to correct our partial sky LG dipole velocity measurement. Now we construct the corrected LG dipole velocity as

$\mathbf{v}_{\mathrm{LG}}(r) \beta^{-1} \equiv \frac{H_{0}}{4 \pi \bar{n}} \frac{1}{N} \sum_{i=1}^{N}\left[\mathbf{D}_{\mathrm{HI}}^{\mathrm{PS}}(r)+\mathbf{X}^{i}(r)\right]$,

correcting both direction and module of the LG dipole velocity for a PS data catalogue, and, for this, appropriate for the ALFALFA catalogue (see Appendix $\mathrm{C}$ for details of this correction procedure).

To obtain a good performance with this correction procedure, one has to restrict the set of FS log-normal simulations to those whose clustering dipole direction are close to the LG velocity direction in CMB frame, $(l, b)=\left(273^{\circ} \pm 3^{\circ}, 29^{\circ} \pm 3^{\circ}\right)$, and for this we consider those maps where the misalignment between both directions is less than $30^{\circ 2}$. The ideal situation would be to consider only simulations where this value is $0^{\circ}$, but this clearly would be a fine tuning, and scientifically invalid, approach. By doing this, we are left with 144 catalogues from a total of 4000 log-normal simulations produced ${ }^{3}$. This is not strange, as already noticed by Kolokotronis et al. (1995), to find suitable simulations that exhibit the main features of our LG universe is a difficult task, which decreases considerably the number of suitable catalogues for analyses.

This dipole correction procedure was done using a set of simulated log-normal catalogues constructed with the public code ${ }^{4}$ presented in Agrawal et al. (2017). The log-normal approach assumes

2 The robustness of our results has been tested for several values of maximum misalignment: $20^{\circ}, 30^{\circ}, 40^{\circ}$, achieving basically the same results for each case.

3 We tested the robustness of this, apparently small, number of catalogues. We first perform the analyses with these 144 catalogues and calculate $f \sigma_{8}$; then we add new 1000 FS log-normal catalogues to the original 4000, selecting a total of 191 catalogues, we redo the analyses and again calculate $f \sigma_{8}$ obtaining the same result.

4 https://bitbucket.org/komatsu5147/lognormal_ galaxies/src/master/
Table 1. Survey configuration and cosmological parameters from the Planck last data release (Planck Collaboration 2020) used to generate the set of $N_{\mathrm{S}}=4000 \log$-normal realizations used in the analyses.

\begin{tabular}{c|c}
\hline Survey configuration & Cosmological parameters \\
\hline$z=0.0$ & $\Omega_{c} h^{2}=0.1202$ \\
$b=1.0$ & $\Sigma m_{\nu}=0.0600$ \\
$N_{g}=2 \times 10^{5}$ & $n_{s}=0.9649$ \\
$L_{x}=230$ & $\ln \left(10 A_{s}\right)=3.045$ \\
$L_{y}=230$ & $\Omega_{b} h^{2}=0.02236$ \\
$L_{z}=230$ & $h=0.6727$ \\
\hline
\end{tabular}

that the matter and galaxy density fields can be represented by a log-normal probability density function. In Agrawal et al. (2017), the authors show the remarkable agreement between the input and measurements for the correlation function and power spectrum, important features used to analyse galaxy clustering in surveys.

In table 1 we show all the input parameters needed to generate our log-normal FS simulated catalogues. In the first column we present the survey configuration: the box dimensions, $L_{x}, L_{y}$, and $L_{z}$, the number of galaxies ${ }^{5}, N_{g}$, the redshift at which we generate the input power spectrum, $z$, and the bias, $b$. The matter power spectrum, $P(k)$, is calculated using Eisenstein \& $\mathrm{Hu}(\mathrm{EH})$ transfer function (Eisenstein \& Hu 1998). The code uses this approach by default, in case one does not provide a table of $P(k)$ values calculated externally (see Appendix D for a check of the accuracy of the EH approach). All these parameters were chosen in order to reproduce the ALFALFA survey. In the second column of table 1 we observe the cosmological parameters, as given by the Planck Collaboration (2020). For the bias choice, see Section 3.3.

It is worth mentioning that, even though the log-normal simulations do not reproduce accurately the velocity field when compared with N-body simulations, as shown by Agrawal et al. (2017) using the linearised continuity equation of the matter fields, our analyses using equations (9) and (10) use only the galaxy positions. Therefore, as our tests suggest in appendix C, the log-normal catalogues can be used to obtain robust results of the ALFALFA dipole convergence and, consequently, an accurate measurement of $f \sigma_{8}$.

Finally, one can calculate the error in equation (13) using the same procedure used to compute the shot-noise error. The covariance matrix due to the partial sky coverage using the log-normal (LN) catalogues can be calculated as

$$
\begin{aligned}
C_{\mathrm{LN}}^{i j}= & \frac{1}{N-1} \sum_{k=1}^{N}\left(\left[v_{\mathrm{LG}}\left(r_{i}\right) \beta^{-1}\right]^{k}-\left\langle\left[v_{\mathrm{LG}}\left(r_{i}\right) \beta^{-1}\right]\right\rangle\right) \times \\
& \left(\left[v_{\mathrm{LG}}\left(r_{j}\right) \beta^{-1}\right]^{k}-\left\langle\left[v_{\mathrm{LG}}\left(r_{j}\right) \beta^{-1}\right]\right\rangle\right)
\end{aligned}
$$

where $v_{\mathrm{LG}} \equiv\left|\mathbf{v}_{\mathrm{LG}}\right|,\left[v_{\mathrm{LG}}(r) \beta^{-1}\right]^{k}$ is the LG velocity for the $k$ th log-normal realization, and $\left\langle\left[v_{\mathrm{LG}}(r) \beta^{-1}\right]\right\rangle$ is the average $\mathrm{LG}$ velocity over $N$ realizations.

Notice that to obtain the errors in both $\beta$ and $f \sigma_{8}$, we combine both covariance matrices, that is,

$C_{\mathrm{SN}+\mathrm{LN}}^{i j}=C_{\mathrm{SN}}^{i j}+C_{\mathrm{LN}}^{i j}$.

5 This number is not constant in all realizations, but their fluctuations around $N=7798$ (the number of HI line sources of the catalogue in analysis) are not significant. 


\subsection{Measuring $\sigma_{8}^{\mathrm{tr}}$}

In order to perform a measurement of $f \sigma_{8}$ in the local Universe using our data sample, we first calculate $\beta$, using equation (9), and, then, $\sigma_{8}^{\mathrm{tr}}$, the matter fluctuation of the HI line sources of our data sample in spheres of $8 \mathrm{Mpc} / \mathrm{h}$. For galaxy samples, $\sigma_{8}^{\text {tr }}$ was observed to be close to 1 (Juszkiewicz et al. 2009; Boruah, Hudson \& Lavaux 2019). However, this result will depend on the bias of the tracer. In the $\Lambda$ CDM context one expects $\sigma_{8}^{\text {tr }}<1$ for $b \lesssim 1$.

In this work we calculate $\sigma_{8}^{\mathrm{tr}}$ using the relationship

$\sigma_{8}^{\mathrm{tr}}=b_{\mathrm{HI}} \sigma_{8}$,

where $\sigma_{8}=0.8120 \pm 0.0073$ from Planck Collaboration $(2020)^{6}$. The linear bias, $b_{\mathrm{HI}}$, for the HI line sources can be obtained from the work of Martin et al. (2012), where they calculated the bias for different scales. In Fig. 10 of this reference we observe, in the interval 3-30 Mpc/h, a fluctuation around 1, where the HI tracer reflects the underlying matter distribution. Then, we take the data points in this scale range and fit a horizontal line to them, obtaining

$b_{\mathrm{HI}}=0.99 \pm 0.11$.

This bias value motivated us to fix $b=1.0$ to generate the lognormal simulations. Using equation (16), we obtain the variance for our sample, that is, $\sigma_{8}^{\mathrm{tr}}=0.80 \pm 0.09$.

\section{RESULTS}

In Fig. 6 we show the result of applying equations (9) and (13) to our ALFALFA sample, where $\bar{n}=0.04 \mathrm{Mpc}^{-3}$ and $H_{0}=67.27$ $\mathrm{km} / \mathrm{s} / \mathrm{Mpc}$ (Planck Collaboration 2020). The error bars come from the diagonal terms of the covariance matrix given by equation (15) (see Fig. 7), accounting for the cosmic variance, that comes from the $144 \log$-normal simulations, using equation (14), and the shotnoise, from the 144 Monte Carlo realizations using equation (11). In the same plot, we compare the corrected (red squares) and the uncorrected (blue triangles) LG velocity. One can see that, in average, for scales smaller than $\sim 70 \mathrm{Mpc}$ the PS uncorrected analysis underestimate the dipole amplitude, with an opposite behaviour for larger scales. The black line and the shaded region represent the convergence value and $1 \sigma$ uncertainty of the LG velocity function, respectively. We describe below how we obtain this value.

To obtain the convergence value, $V$, taking into account the error bars, we performed a numerical derivative of the LG velocity function, that is,

$\frac{d}{d r} \mathbf{v}_{\mathrm{LG}}(r) \beta^{-1}=\frac{H_{0}}{4 \pi \bar{n}} \frac{1}{N} \sum_{i=1}^{N} \frac{d}{d r}\left[\mathbf{D}_{\mathrm{HI}}^{\mathrm{PS}}(r)+\mathbf{X}^{i}(r)\right]$,

looking for the scale interval consistent with zero, i.e., where this function attains a maximum, ensuring an accurate measurement of this convergence value. In Fig. 8 we show the result of equation (18) applied to the ALFALFA LG velocity, the $1 \sigma$ error bars are estimated performing the same sequence of analyses over each of the 144 simulations. We observe that the derivative is consistent with zero, within a $1 \sigma$ confidence level, in the interval $45-63 \mathrm{Mpc}$. Thus, to obtain the convergence value, we take the LG velocity data

6 This value of $\sigma_{8}$ corresponds to $z=0$; assuming the $\Lambda$ CDM fiducial model of table 1 one finds: $\sigma_{8}(z=0)-\sigma_{8}(z=0.013)=0.0056$, a difference smaller than the error bar that does not modify our main result. For this, we use $\sigma_{8}(z=0.013) \simeq \sigma_{8}(z=0)=0.8120 \pm 0.0073$.

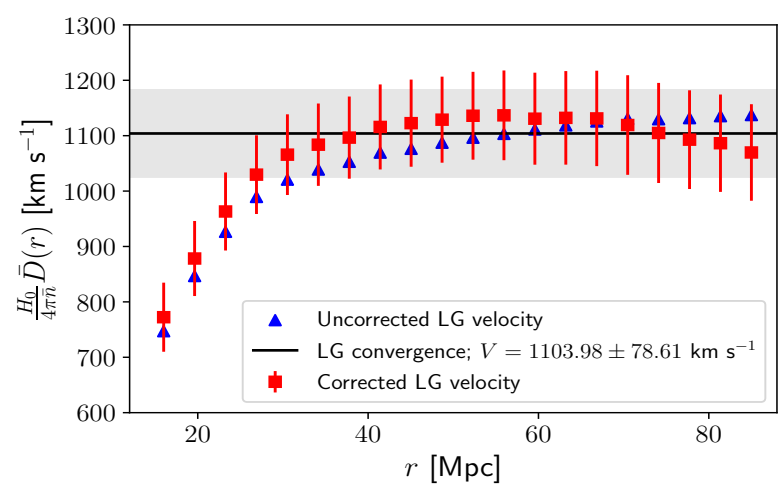

Figure 6. The ALFALFA LG velocity function, in real space, as a function of the radial distance from the observer to a surface of radius $r$, where the dipole is evaluated. The error bars take into account cosmic variance and shot-noise errors (see the text for details). The convergence value, $V$, and its $1 \sigma$ error are represented by the black horizontal line and the shaded region, respectively.

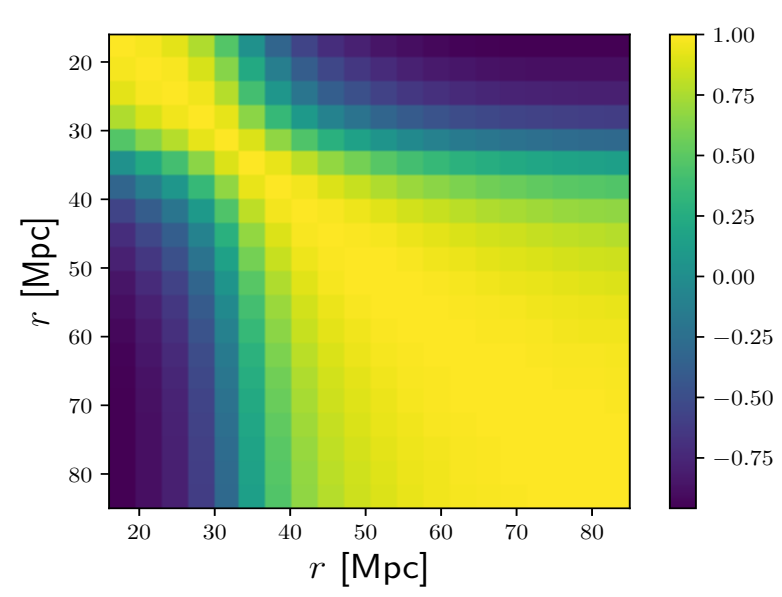

Figure 7. Reduced covariance matrix, $C^{i j} / \sqrt{C^{i i} \times C^{j j}}$, obtained from equation (15). The covariance matrix is obtained combining the shot-noise error, using Monte Carlo realizations, and the error due to the correction for partial sky survey, using log-normal simulations.

points in this interval and fit for a horizontal line to them, obtaining

$$
V=1103.98 \pm 78.61 \mathrm{~km} / \mathrm{s}
$$

In the Fig. 6, we observe that the ALFALFA corrected LG velocity is consistent with this convergence value (black vertical line), within the $1 \sigma$ level, until $85 \mathrm{Mpc}$, i.e., the limit of our analysis. As our selection function is of order $\phi(r=85 \mathrm{Mpc}) \simeq 0.3$, we are confident that our result does not indicate an artificial convergence, as discussed in Scaramella, Vettolani \& Zamorani (1994).

Then, one can find the velocity scale parameter $\beta$ from equation (9) by using $V=1103.98 \pm 78.61 \mathrm{~km} / \mathrm{s}$ value and the known LG peculiar velocity in the CMB frame $u_{\mathrm{LG}} \equiv\left|\mathbf{u}_{\mathrm{LG}}\right|=627 \pm 22$ km/s (Courteau \& Van Den Bergh 1999; Erdoğdu et al. 2006)

$\beta=\frac{u_{\mathrm{LG}}}{V}=0.57 \pm 0.04$.

Because $f=b \beta$, this measurement of $\beta$ combined with the bias $b$ of the sample in analysis, equation (17), provides a measurement 


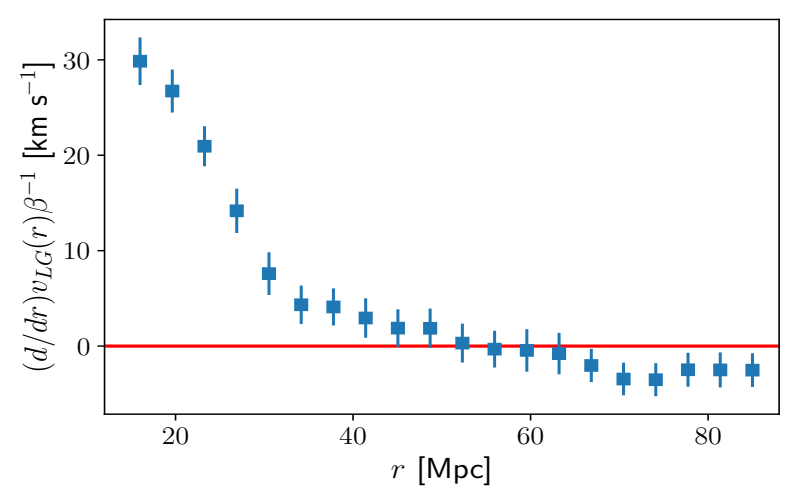

Figure 8. Numerical derivative of the ALFALFA LG velocity function (red squares in Fig. 6) with respect to the radial distance $r$. The error bars correspond to the $1 \sigma$ dispersion from the $144 \log$-normal simulations.

of the growth rate of structures $f$,

$f=0.56 \pm 0.07$,

at $\bar{z}=0.013$.

Our measurement of $\beta$ shows a good agreement with the value $\beta^{\Lambda \mathrm{CDM}}$ expected in the $\Lambda \mathrm{CDM}$ model. In fact, equation (2) with $\gamma=0.55$ and $\Omega_{m}=0.3150$ (from table 1) gives $f^{\Lambda \mathrm{CDM}}=0.54$ at $\bar{z}=0.013$; then, using $b_{\mathrm{HI}}=0.99 \pm 0.11$ we have $\beta^{\Lambda \mathrm{CDM}}=$ $0.54 \pm 0.06$.

Finally, we can obtain our main result $f \sigma_{8}$; for this we use equation (3) to combine $\beta$, from equation (20), with $\sigma_{8}^{\text {tr }}=0.80 \pm$ 0.09 , obtained using equations (16) and (17), to get

$f \sigma_{8}=0.46 \pm 0.06$

at $\bar{z}=0.013$, consistent with the $\Lambda \mathrm{CDM}$ model at $1 \sigma$ confidence level, $\left[f \sigma_{8}\right]^{\Lambda \mathrm{CDM}}=0.43 \pm 0.02$. In Fig. 9 we display, for comparison, our result together with a sample of measurements of $f \sigma_{8}$ at low redshift, performed through diverse methodologies that analyse several cosmological tracers.

As a robustness test for the $H_{0}$ value, a parameter in the calculations of the LG dipole velocity, we have produced another set of log-normal simulations with the hypothesis $H_{0}=74.03$ $\mathrm{km} / \mathrm{s} / \mathrm{Mpc}$ (Riess et al. 2019) (i.e., $h=0.7403$ ). We have repeated the analyses finding: $\beta=0.51 \pm 0.02$ and $f \sigma_{8}=0.41 \pm 0.05$, which reproduces, within $1 \sigma$, the results already obtained. This reveals that the value of the Hubble constant $H_{0}$ has a limited impact on our analyses, and that our results are robust under different values of $H_{0}$ reported in the literature.

\section{CONCLUSIONS}

The structures growth data of the observed Universe has the potential to differentiate between the metric theory that supports the standard cosmological model, that is GR theory, from those based on modified gravity models. Due to this scenario, efforts are being done to analyse several cosmological tracers with diverse approaches and methodologies. One of these, is the gravitational dipole technique (Hudson 1993; Scaramella, Vettolani \& Zamorani 1994). In summary, this methodology compares the peculiar velocity of the LG of galaxies, inferred from the CMB dipole, to the LG gravitational acceleration calculated from a given cosmological tracer. Using the catalogue of extra-galactic HI line sources

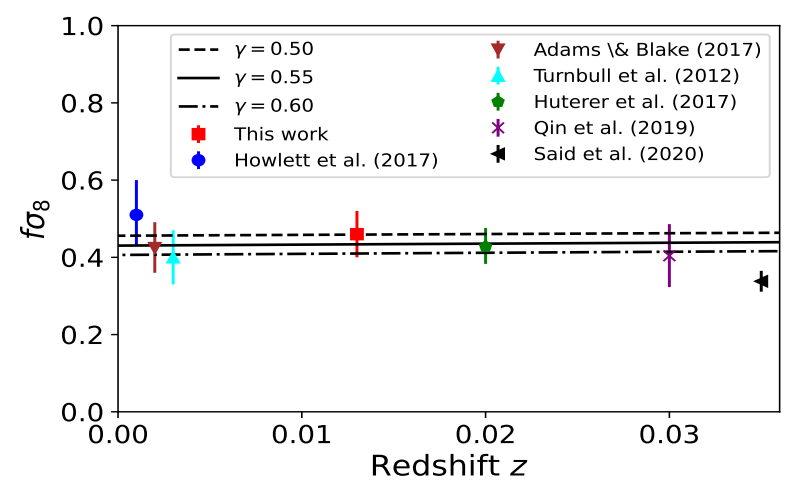

Figure 9. Measurements of $f \sigma_{8}$ for the local Universe, where our result is shown as a red square. We observe a good agreement with the $\Lambda$ CDM model, corresponding to the case $\gamma=0.55$ (see equation (2)). The blue dot is the work of Howlett et al. (2017), using the 2MTF galaxies, and the inverted brown triangle is the outcome of Adam \& Blake (2017) for the $6 \mathrm{dF}$ galaxy survey data. The work of Turnbull et al. (2012), cyan triangle, used a compilation of SNe Ia. These three measurements are slightly shifted to the right for a better visualization, but they were calculated at $z \simeq 0$. The green pentagon shows the result of Huterer et al. (2017) combining low redshift SNe Ia with the $6 \mathrm{dF}$ galaxy survey. The last two points correspond to Qin, Howlett, \& Staveley-Smith (2019) (who combine the 2MTF and $6 \mathrm{dF}$ galaxies) and Said et al. (2020) (who performed joint analyses of the 6dFGS and SDSS data) at $z=0.03$ and $z=0.035$, respectively.

provided by the ALFALFA survey (Haynes et al. 2018), we investigate the growth rate of cosmic structures in the local Universe.

In fact, these analyses can be biased by various systematic effects, such as shot-noise, RSD, and non-linear effects, as well as the incomplete sky coverage of the survey. As discussed in Section 3.2, the latter is the main source of systematics in our analyses. To correct the LG velocity dipole for the effect produced by the fact that the observed data in the ALFALFA survey covers a partial region of the celestial sphere, instead of the full sky, we use sets of FS and PS log-normal simulations according to the correction procedure described in Section 3.2.2. In contrast, while the RSD effect can be avoided, since our data sample allows the dipole calculation in real space, the shot-noise contributes with a relatively small error due to the high number density of the sample, as shown in the analyses performed with the set of Monte Carlo realizations.

Additionally, our analyses show that it is possible to estimate the product of the growth rate and the matter fluctuation, $f \sigma_{8}$, through the gravitational dipole approach (Strauss \& Willick 1995; Scaramella, Vettolani \& Zamorani 1994) using a partial sky catalogue, as long as the bias of the cosmological tracer and the correction due to the partial sky survey are carefully taken into account. This way, we found that the magnitude of the dipole velocity calculated from the ALFALFA sample reaches the convergence around $60 \mathrm{Mpc}$, and its magnitude leads to our estimate of the velocity scale parameter, $\beta=0.57 \pm 0.04$. Together with our measurement of the matter fluctuation in the local Universe, $\sigma_{8}^{\mathrm{tr}}=0.80 \pm 0.09$, it provides the value $f \sigma_{8}=0.46 \pm 0.06$ at $\bar{z}=0.013$. This measurement is in good agreement, at $1 \sigma$ level, with the value obtained in the $\Lambda \mathrm{CDM}$ concordance model: $\left[f \sigma_{8}\right]^{\Lambda \mathrm{CDM}}=0.43 \pm 0.02$. As observed in Fig. 9, where we show a small compilation of $f \sigma_{8}$ values in the local Universe, our result is in good consonance with measurements obtained analysing several cosmological tracers through methodologies different from ours. Furthermore, our analyses of the ALFALFA sample also provide a measurement of the growth rate of structures $f=0.56 \pm 0.07$, at $\bar{z}=0.013$. 


\section{ACKNOWLEDGEMENTS}

The authors thank PROPG-CAPES/FAPEAM program, CNPq, CAPES, and FAPESP (process no. 2019/06040-0) for the grants under which this work was carried out.

\section{DATA AVAILABILITY}

The data underlying this article were accessed from http:// egg.astro.cornell.edu/alfalfa/data/index.php. The derived data generated in this research will be shared on reasonable request to the corresponding author.

\section{REFERENCES}

Abbott, T. M. C., et al., 2018, Phys. Rev. D, 98, 043526, [arXiv:1708.01530] Adams, C., \& Blake, C., 2017, MNRAS, 471, 839, [arXiv:1706.05205]

Agrawal, A., et al., 2017, J. Cosmol. Astropart. P., 10, 003, [arXiv:1706.09195]

Alam, S., et al., 2017, MNRAS, 470, 2617, [arXiv:1607.03155]

Aubert, M., et al., 2020, [preprint arXiv:2007.09013]

Avila, F., et al., 2018, J. Cosmol. Astropart. P., 12, 041, [arXiv:1806.04541]

Avila, F., et al., 2019, MNRAS, 488, 1481, [arXiv:1906.10744]

Basilakos, S., \& Plionis, M., 1998, MNRAS, 299, 637, [astro-ph/9801271]

Basilakos, S., \& Plionis, M., 2006, MNRAS, 373, 1112, [astro-ph/0609476]

Basilakos, S., 2012, Int. J. Mod. Phys. D, 21, 1250064, [arXiv:1202.1637]

Bautista, J. E., et al., 2018, ApJ, 863, 110, [arXiv:1712.08064]

Bilicki, M., et al., 2011, ApJ, 741, 31, [arXiv:1102.4356]

Bilicki, M., 2012, [preprint 1205.1970v1]

Boruah, S. S., Hudson, M. J., \& Lavaux, G., 2019, [preprint arXiv: 1912.09383]

Courteau, S., \& Van Den Bergh, S, 1999, AJ, 118, 337, [astro-ph/9903298]

de Carvalho, E., et al., 2018, J. Cosmol. Astropart. P., 04, 064, [arXiv:1709.00113]

de Carvalho, E., et al., 2020, MNRAS, 492, 4469, [arXiv:2002.01109]

de Carvalho, E., et al., 2021, A\&A, 649, A20, [arXiv:2103.14121]

Eisenstein, D. J., \& Hu, W., 1998, ApJ, 496, 605, [arXiv:astro-ph/9709112]

Erdoğdu, P., et al., 2006, MNRAS, 368, 1515, [astro-ph/0507166]

Giovanelli, R., \& Haynes, M. P., 2016, Astron Astrophys Rev, 24, 1, [arXiv:1510.04660]

Haynes, M. P., et al., 2011, AJ, 142, 170, [arXiv:1109.0027]

Haynes, M. P., et al., 2018, ApJ, 861, 49, [arXiv:1805.11499]

Haude, S., et al., 2019, [preprint 1912.04560]

Heinesen, A., 2020, J. Cosmol. Astropart. P., 10, 052, [arXiv:2006.15022]

Howlett, C., et al., 2017, MNRAS, 471, 3135, [arXiv:1706.05130]

Hudson, M. J., 1993, MNRAS, 265, 72

Huterer, D., et al., 2017, J. Cosmol. Astropart. P., 5, 015, [arXiv:1611.09862]

Jones, M. G., et al., 2016, MNRAS, 457, 4393, [arXiv:1510.07050]

Jones, M. G., et al., 2018, MNRAS, 477, 2, [arXiv:1802.00053]

Jones, M. G., et al., 2020, MNRAS, 494, 2090, [arXiv:2003.09302]

Juszkiewicz, R., et al., 2009, J. Cosmol. Astropart. P., 2, 021, [arXiv:0901.0697v3]

Kaiser, N., 1987, MNRAS, 227, 1

Kaiser, N., \& Lahav, O., 1989, MNRAS, 237, 129

Kocevski, D. D., \& Ebeling, H., 2006, ApJ, 645, 1043, [astro-ph/0510106]

Kogut, A., et al., 1993, ApJ, 419, 1, [astro-ph/9312056]

Kolokotronis, V., et al., 1996, MNRAS, 280, 186, [arXiv:astro-ph/9511053]

Lewis, A., Challinor, A., \& Lasenby, A., 2000, ApJ, 538, 473, [arXiv:astro$\mathrm{ph} / 9911177]$

Linder, E. V., \& Cahn, R. N., 2007, Astropart. Phys., 28, 481, [astro$\mathrm{ph} / 0701317 \mathrm{v} 2]$

Marques, G. A., Novaes, C. P., Bernui A., Ferreira, I. S., 2018, MNRAS, 473, 165, [arXiv:1708.09793]

Marques, G. A., \& Bernui, A., 2020, J. Cosmol. Astropart. P., 5, 052, [arXiv: 1908.04854]
Martin, A. M., et al., 2012, ApJ, 750, 38, [arXiv:1202.6005v1]

Master, K, L., 2005, PhD thesis, Univ. Cornell

Nunes, R. C., \& Bernui, A., 2020, Eur. Phys. J. C, 80, 1025 , [arXiv:2008.03259]

O’Donoghue, A. A., et al., 2018, [preprint arXiv:1811.01283]

Pandey, B., \& Sarkar, S., 2020, MNRAS, 498, 6069, [arXiv:2002.08400]

Papageorgiou, A., et al., MNRAS, 2012, 422, 106, [arXiv:1201.4878]

Papastergis, E., et al., 2013, ApJ, 776, 43, [arXiv:1308.2661v1]

Peebles, P. J. E., 1980, The large-scale structure of the universe. Princeton Univ. Press, Princeton, NJ

Pezzotta, A., et al., 2017, A\&A, 604, A33, [arXiv:1612.05645]

Planck Collaboration, 2016, A\&A, 594, A24, [arXiv:1502.01597]

Planck Collaboration, 2020, A\&A, 641, A6, [preprint, arXiv:1807.06209]

Qin, F., Howlett, C., \& Staveley-Smith, L., 2019, MNRAS, 487, 5235, [arXiv:1906.02874]

Riess, A. G., et al., 2019, ApJ, 876, 85, [arXiv:1903.07603]

Rowan-Robinson, M., et al., 2000, MNRAS, 314, 375, [arXiv:astro$\mathrm{ph} / 9912223$ ]

Said, K., et al., 2020, MNRAS, 497, 1275, [arXiv:2007.04993]

Sarkar, S., \& Pandey, B., 2019, MNRAS, 485, 4743, [arXiv:1812.03661]

Scaramella, R., Vettolani, G., \& Zamorani, G., 1994, ApJ, 422, 1

Schmoldt, I., et al., 1999, MNRAS, 304, 893, [astro-ph/9901087]

Springob, C. M., et al., 2007, ApJS, 172, 599, [arXiv:0705.0647]

Strauss, M. A., \& Willick, J. A., 1995, Phys. Rep., 261, 271, [astro$\mathrm{ph} / 9502079]$

Turnbull, S. J., 2012, MNRAS, 420, 447, [arXiv:1111.0631]

Van den Bergh, S., 2000, The galaxies of the Local Group. Cambridge Univ. Press, Cambridge, UK

Van der Marel, R. P., \& Guhathakurta, P., 2008, ApJ, 678, 187, [arXiv:0709.3747]

\section{APPENDIX A: TESTING THE IMPACT OF DISTANCE UNCERTAINTIES ON DIPOLE ANALYSES}

In this appendix we show that the possible underestimation in the measurements of the distance errors in the ALFALFA catalogue produces a negligible effect in our dipole analyses. To show this we perform the following test. Consider our ALFALFA data set of $N=7798$ distance values: $\left\{d_{i}\right\}, i=1,2, \cdots, 7798$. We generate 4000 Monte Carlo realizations, where each realization contains 7798 simulated distance values, the $i$ th distance $d_{i}^{\text {sim }}$ is taken from a normal distribution with mean value $d_{i}$ (the true value) and standard deviation $\sigma_{i}=0.2 d_{i}$. That is, we consider Monte Carlo realizations as simulated catalogues with 'wrong' distance values, quantities that deviates in $20 \%$ on average from the original 'true' values given in the ALFALFA catalogue, a conservative deviation as suggested by the information contained in Boruah, Hudson \& Lavaux (2019).

In the upper panel of Fig. A1, we show the result of this test. The squares represent the data values obtained analysing the dipole value for the ALFALFA catalogue, and the error bars correspond to the standard deviation for the same analysis done with each one of the 4000 Monte Carlo realizations. According to this test, the assumed deviations in the distance values have a negligible effect on the determination of the dipole, as observed in the lower panel of Fig. A1, where one can see that the error bars correspond to less than $5 \%$ of the measured dipole amplitude.

\section{APPENDIX B: TEST FOR PECULIAR VELOCITIES}

From Fig. 1 one observes that some HI line sources deviate largely from the Hubble flow, indicating that these objects undergo strong gravitational interactions in the local Universe, as a consequence 

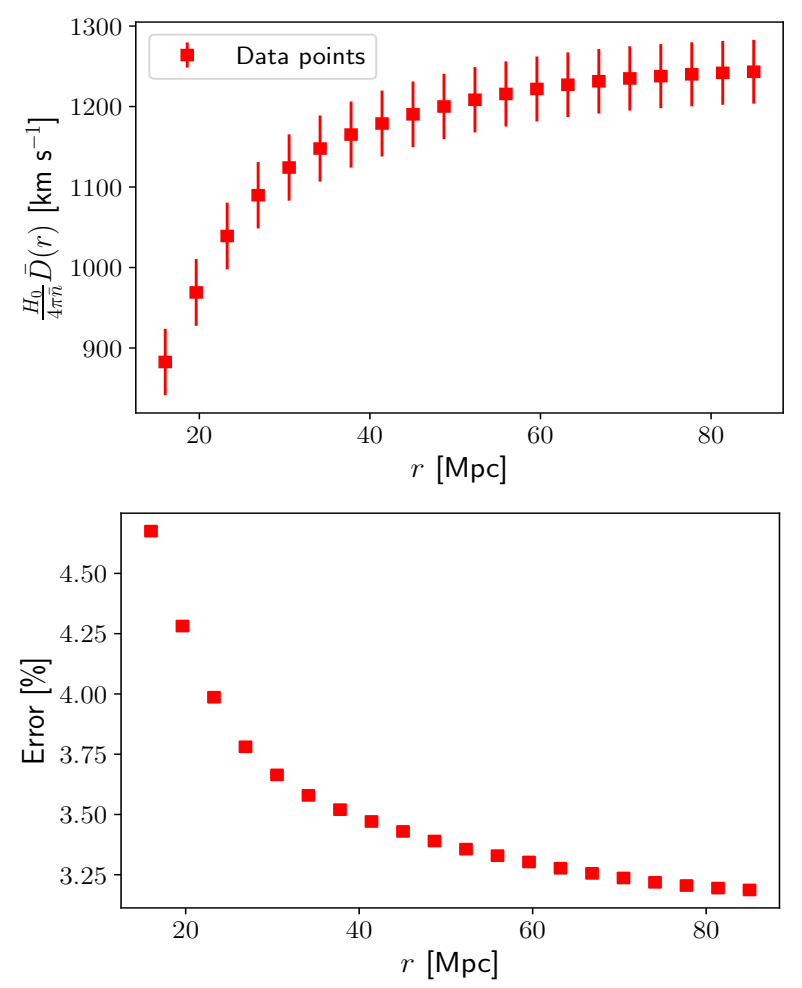

Figure A1. Upper panel: Dipole analyses of the ALFALFA catalogue with error bars given by 4000 Monte Carlo realizations generated imposing large artificial errors in distance measurements. Lower panel: Error, in percentage, with respect to the dipole amplitude. See the text for details.

they have large peculiar velocities. To evaluate if a set of large peculiar velocities can affect our dipole measurement we performed a test. We calculate, for this set of $N=7798 \mathrm{HI}$ line sources, the $1 \sigma$ dispersion of velocities compared with the velocity expected in the Hubble flow, obtaining $325 \mathrm{~km} / \mathrm{s}$, represented by red lines in the upper panel of Fig. B1. After that, we remove from the sample those objects with velocities out of this $1 \sigma$ dispersion level, remaining a test sample of $N^{\text {test }}=6881 \mathrm{HI}$ line sources. We then compute the LG velocities for each of these two samples: with $N=7798$ and with $N^{\text {test }}=6881$ objects. In the lower panel of Fig. B1 we compute the relative difference between these uncorrected LG velocities, which shows a maximum deviation of $\sim 3 \%$. We further investigate the impact of these objects with large peculiar velocities in the measurement of the corrected LG velocity. Our result shows that these uncorrected LG velocities are within the $1 \sigma$ error of the corrected LG velocity. Therefore, we conclude that the effect caused by the peculiar velocities in our analyses is negligible, and their impact is within the error of our dipole measurement.

\section{APPENDIX C: THE CORRECTION PROCEDURE OF THE LG DIPOLE VELOCITY}

With the procedure used in section 3.2.2 we correct the partial sky clustering dipole measurement, $\mathbf{D}_{\text {data }}^{\mathrm{PS}}(r)$, as

$\mathbf{D}_{\text {data }}^{\text {corrected }, i}(r)=\mathbf{D}_{\text {data }}^{\mathrm{PS}}(r)+\mathbf{X}^{i}(r)$,

$i=1,2, \cdots, N$, where $N$ is the number of simulated catalogues used in the correction analyses. The correction term, $\mathbf{X}^{i}(r)$ defined in equation (12), is the vectorial difference between the full sky
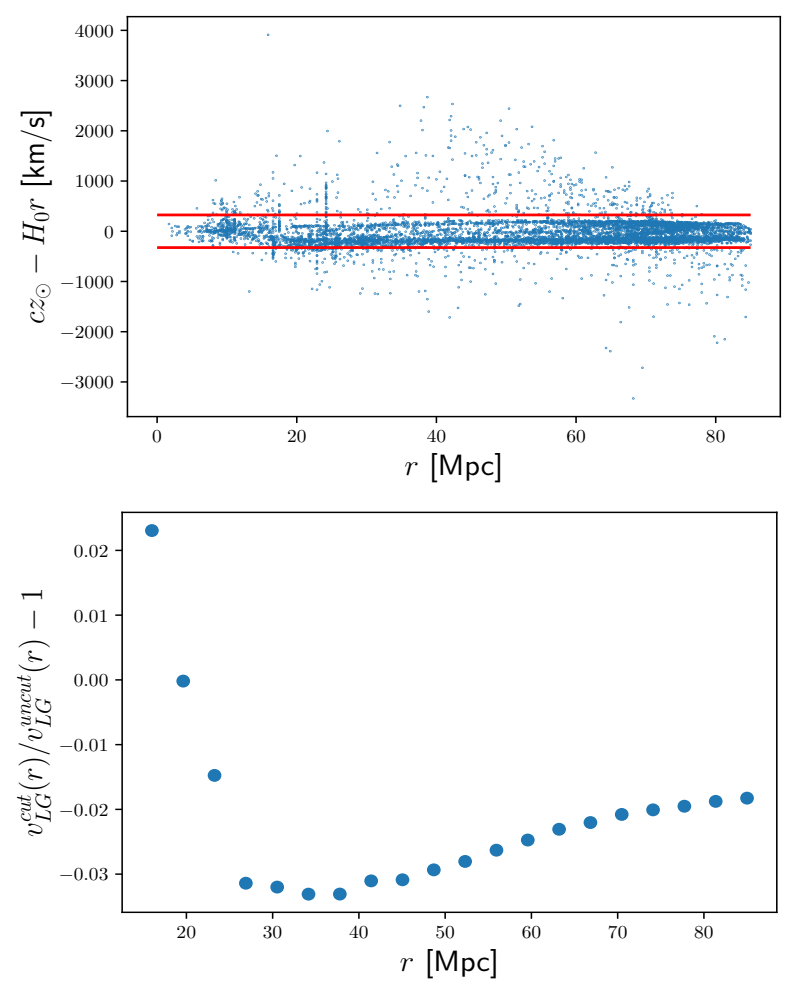

Figure B1. Upper panel: Velocities of the ALFALFA HI line sources with respect to the Hubble flow within (i.e., inside the red lines) and out (i.e., outside the red lines) $1 \sigma$ dispersion of the Hubble flow. Lower panel: Relative difference of the uncorrected LG velocity of the test sample, obtained removing the cosmic objects with large peculiar velocities $\left(v_{L G}^{c u t}\right)$, with respect to the uncorrected LG velocity of the original sample $\left(v_{L G}^{\text {uncut }}\right)$.

(FS) and partial sky (PS) clustering dipoles obtained from a set of $N$ log-normal simulations, for $i=1, \cdots, N$. In equation (C1), the vector $\mathbf{X}^{i}(r)$ contains the information loosed due to the partial sky coverage of the data survey. Thus, this procedure produces, for each radial distance $r$, a set of $N$ values $\left\{\mathrm{D}^{\text {corrected, } i}\right\}=\left\{\left|\mathbf{D}^{\text {corrected, } i}\right|\right\}$ that we use to find the correct clustering dipole. The corrected clustering LG dipole $\overline{\mathrm{D}}^{\text {corrected }}(r)$ is the average of the set of $N$ values: $\left\{\mathrm{D}^{\text {corrected, } i}(r)\right\}$, and the associated error is the standard deviation of this set. These data, $\frac{H_{0}}{4 \pi \bar{n}} \overline{\mathrm{D}}^{\text {corrected }}(r)$, are plotted as red squares in Fig. 6, while the blue triangles correspond to the partial sky uncorrected data $\frac{H_{0}}{4 \pi \bar{n}} \mathrm{D}_{\text {data }}^{\text {PS }}(r)$.

Finally, the corrected clustering dipole, $\overline{\mathrm{D}}^{\text {corrected }}(r)$, is related to the $\mathrm{LG}$ velocity, $\mathrm{v}_{\mathrm{LG}}(r)$, through $\beta$ as

$\mathrm{v}_{\mathrm{LG}}(r) \beta^{-1}=\frac{H_{0}}{4 \pi \bar{n}} \overline{\mathrm{D}}^{\text {corrected }}(r)$,

equivalent to the equation (13)

A robustness test is in due here, to show the performance of this correction procedure. Firstly, we select the set of $N$ FS lognormal simulated catalogues where the misalignment of their clustering dipole and the CMB dipole direction is less than $30^{\circ}$. Then we have two sets of simulated catalogues: $N$ FS log-normal maps, and $N$ PS log-normal maps (obtained from the first set after applying the ALFALFA footprint). From this set of PS catalogues, we select one of them to be considered the data catalogue. The remaining $N-1$ PS catalogues and the $N-1$ FS catalogues will be used in the correction procedure of this data catalogue.

The second step is to calculate the uncorrected and the cor- 


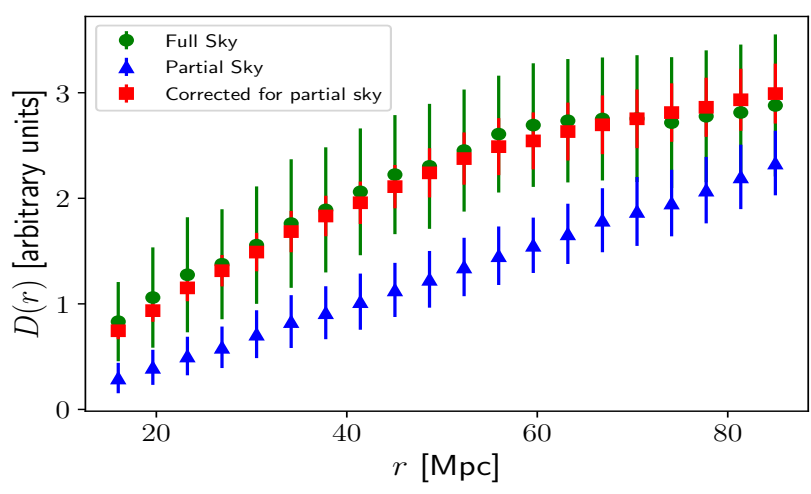

Figure C1. Robustness test of the correction procedure, performed for $N=144 \log$-normal catalogues. The results show an excellent agreement between the corrected clustering dipoles for the PS catalogues (red squares) as compared with the clustering dipoles of the corresponding FS catalogues (green dots). The average of the uncorrected PS dipoles is represented by blue triangles.

rected clustering dipoles of this data catalogue according to equations (12) and (C1). After that, we repeat these calculations using each one of the other $N-1$ simulations as the data catalogue.

In the third step we perform the average of these $N$ uncorrected and $N$ corrected clustering dipoles and plot them in Fig. C1. To complete the test one has to calculate the clustering dipole of the $N$ FS catalogues, take their average and plot together with the above data. One clearly observes in Fig. $\mathrm{C} 1$ that our procedure to correct the clustering dipole of the PS simulated catalogues perfectly reproduces the true result.

As a complementary verification, we also test our correction procedure by calculating the misalignment, $\Delta \theta(r)$, as a function of the radial distance, between the LG velocity relative to the CMB frame and the clustering dipole measured from the ALFALFA catalogue. The expected behaviour for $\Delta \theta(r)$ is a decreasing function for large distances, achieving a convergence that depends on the size and location of the data sample on the sky, besides of the deepness of the catalogue (see section 5 of Bilicki et al. (2011)).

We perform the calculation of $\Delta \theta(r)$ with two datasets: the original or uncorrected LG velocity and the corrected LG velocity obtained according to our correction procedure described above. Our results are shown in Fig. C2, where we observe the uncorrected (blue triangles) and the corrected (red squares) $\Delta \theta$ as a function of the radial distance, $r$. For the uncorrected $L G$ velocity data, the misalignment varies between $60^{\circ}$ and $70^{\circ}$, increasing at large scales, very different from a decreasing expected behaviour. For the corrected $L G$ velocity data, the misalignment decreases and at large scales converges to $\sim 45^{\circ}$.

\section{APPENDIX D: TESTING THE EISENSTEIN \& HU APPROACH}

The code of Agrawal et al. (2017), used here to produce the lognormal simulations, calculates the matter power spectrum, $P(k)$, using the Eisenstein \& $\mathrm{Hu}(\mathrm{EH})$ transfer function (Eisenstein \& $\mathrm{Hu}$ 1998). We find interesting to perform a test to check the accuracy of the EH approach compared with the result obtained us-

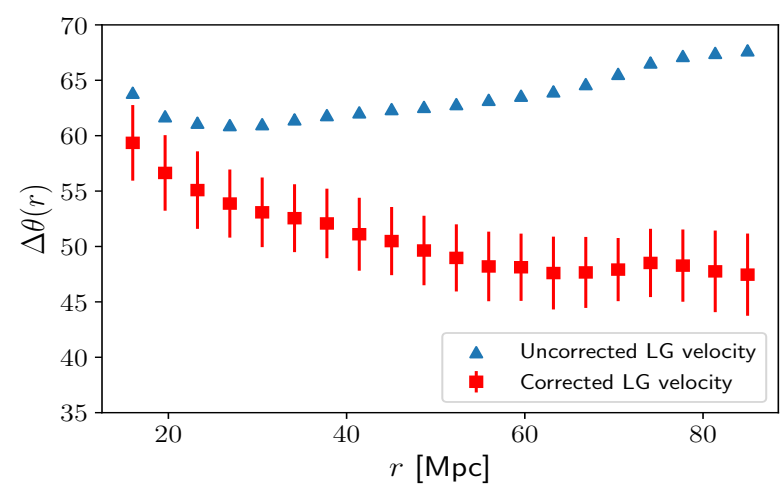

Figure C2. Misalignment between the LG velocity relative to the CMB frame, $\mathbf{u}_{\mathrm{LG}}$, and the clustering dipole measured from the ALFALFA catalogue, $\mathbf{v}_{\mathrm{LG}}(r) \beta^{-1}$, for both cases, the corrected (red squares) and uncorrected (blue triangles) velocities.

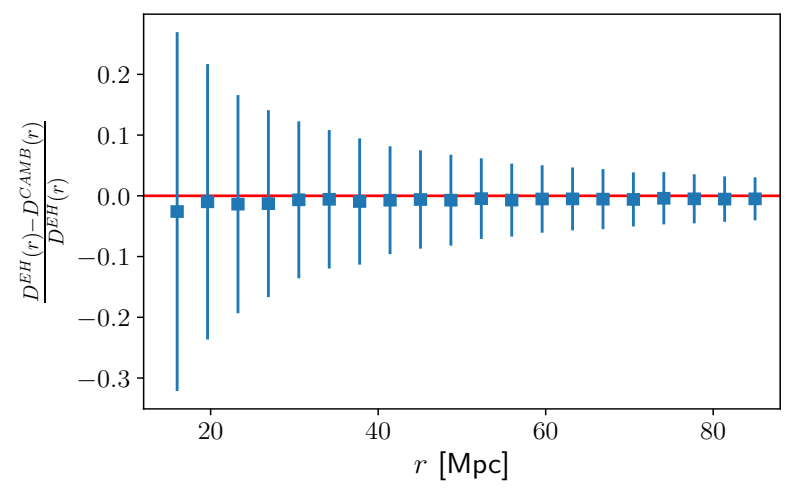

Figure D1. Relative difference of the LG dipole calculated from 1000 log-normal simulations generated using the power spectrum from two approaches: EH (Eisenstein \& Hu 1998) and CAMB (Lewis, Challinor \& Cahn 2000). The horizontal axis refers to the radial distance from the observer to a surface of radius $r$, where the dipole is calculated.

ing the CAMB $\operatorname{code}^{7}$ (Lewis, Challinor \& Cahn 2000), one of the most known and tested Boltzmann codes. For this, first we produce a set of $1000 \log$-normal simulations using the whole pipeline of the code, this includes the internal use of the EH fitting to obtain the matter power spectrum. Second, we generate a set of 1000 log-normal simulations, but this time the matter power spectrum is produced with the CAMB code and introduced into the code as a numerical table (this is an option of the code). Then we perform dipole clustering analyses with both sets of log-normal simulations, our results are shown in Fig. D1 and confirm that both approaches provides the same output.

This paper has been typeset from a $\mathrm{T}_{\mathrm{E}} \mathrm{X} / \mathrm{L} \mathrm{T} \mathrm{E} \mathrm{X}$ file prepared by the author.

\footnotetext{
7 https://camb.info/
} 